\title{
II. Der politische Werdegang eines französischen Liberalen: Paul Reynaud (1878-1966)
}

Paul Reynaud wurde am 15. Oktober 1878 im südfranzösischen Barcelonnette (Département Basses-Alpes ${ }^{1}$ ) als zweites von vier Kindern einer wohlhabenden Familie des mittleren Bürgertums geboren. Sein Vater, Alexandre Reynaud, der einfachen bäuerlichen Verhältnissen entstammte, war als Siebzehnjähriger im Jahre 1857, unter dem Druck wirtschaftlicher Not und einer lokalen Tradition folgend, nach Mexiko ausgewandert und hatte sich dort als Geschäftsmann ein nicht unbedeutendes Vermögen erworben. Dabei waren ihm zweifellos die persönlichen Verbindungen zugute gekommen, die seinen Heimatort mit dem überseeischen Land verknüpften: Seit den zwanziger Jahren des 19. Jahrhunderts bestand in Mexiko eine französische Kolonie, die sich im wesentlichen aus „Barcelonnettes" zusammensetzte und erfolgreich in der Getränke-, Textil- und Papierproduktion betätigte. ${ }^{2}$ In die Heimat zurückgekehrt, konsolidierte Alexandre Reynaud seinen gesellschaftlichen Aufstieg durch die Heirat mit Amélie Gassier, der Tochter eines einflußreichen und wohlhabenden ortsansässigen Bankiers und Lokalpolitikers. Damit hatte er familiäre Bande mit einer der bedeutendsten Notabelnfamilien der Region angeknüpft: Selbst Sohn eines langjährigen Bürgermeisters des Gebirgsdorfes St. Paul, zählte Alexandre Reynaud nun mit seinem Schwiegervater, dem Bürgermeister und Conseiller Général von Barcelonnette, Hippolyte Gassier, sowie dessen Söhnen, dem Conseiller Général Adrien Gassier und dem Abgeordneten und späteren Senator des Départements Basses-Alpes, Aimé Gassier, einige der einflußreichsten Politiker der Gegend zu seinem engsten Familienkreis.

Streng, ernst und nüchtern, aber auch großzügig und rechtschaffen - so beschreibt Paul Reynaud im Rückblick seinen Vater, dem die Familie mit Bewunderung, doch nicht ohne Furcht begegnet sei. Die Mutter, klösterlich erzogen und einer jansenistischen Lebenssicht zuneigend, scheint nur geringen Einfluß auf die Entscheidungen des dominierenden Vaters genommen und, wie dieser, ein nur wenig vertrauliches Verhältnis zu ihren Kindern unterhalten zu haben. ${ }^{3}$ Nach einigen in Nizza verbrachten Wintern verlegte die Familie - der Sohn Paul war fünf Jahre alt - ihren Wohnsitz dauerhaft nach Paris, wo sie sich im bürgerlichen fünften Arrondissement, nicht weit von Panthéon und Jardin du Luxembourg ent-

I Im Jahre 1979 wurde dieses Département in „Alpes-de-Haute-Provence“ umbenannt.

2 Vgl. hierzu Raymonde Antiq-Auvaro, L'émigration des Barcelonnettes au Mexique, Nice 1992; François Arnaud u. a., Les Barcelonnettes au Mexique. Récits et témoignages, Barcelonnette ${ }^{3} 1994$;

Paul Reynaud, Mémoires. Band I: Venu de ma montagne, Paris 1960, S. 23-26. Nach Angaben Paul Reynauds hatte sein Vater mehrere Brüder seiner späteren Frau Amélie Gassier bereits in Mexiko kennengelernt (Reynaud, Mémoires I, S. 27).

3 Reynaud, Mémoires I, S. 26-39. 
fernt, niederließ. Man legte nun Wert auf einen standesgemäßen Lebensstil, der die sorgfältige Ausbildung der vier Kinder einschloß. Zusammen mit seinem älteren Bruder besuchte Paul Reynaud den angesehenen Lycée Montaigne, dann die katholische Ecole Bossuet und den Lycée Louis-le-Grand. Die Mutter, die insbesondere während der regelmäßigen mehrmonatigen Geschäftsreisen des Vaters nach Mexiko die Hauptlast der Erziehung wahrnahm, brachte ihren Kindern den bürgerlichen Bildungskanon nahe: Klavierspiel, Lektüre, Kunstbetrachtung.

Entgegen dem Wunsch des Vaters, der den Eintritt des Sohnes in sein Unternehmen erhofft hatte, entschied sich Paul Reynaud nach bestandenem Baccalaureat für das Rechtsstudium, akzeptierte jedoch die väterliche Bedingung, während der beiden ersten Studienjahre ab Oktober 1896 gleichzeitig an der „Ecole des Hautes Etudes Commerciales“ Kurse zu besuchen. Als 64. von 118 Jahrgangsgenossen beendete er seine dortige Ausbildung im Juli 1898 mit der Gesamtnote "Assez bien“. ${ }^{4}$ Nachdem er im Juli 1899 die Licence en droit ${ }^{5}$ erworben hatte eine erste Voraussetzung für die Zulassung zum Anwaltsberuf ${ }^{6}$ - leistete Reynaud beim 37. Infanterieregiment in Nancy seinen zweijährigen Wehrdienst ab, nach eigenem Bekunden ohne größeren Widerwillen.?

Im März 1901 als „avocat stagiaire" 8 bei der Pariser Anwaltskammer zugelassen, unterbrach Reynaud schon sehr bald ein erstes Mal seine „stage“, um zwischen Januar 1903 und Januar 1907 in mehreren Pariser Anwaltsbüros als Praktikant, schließlich als Kanzleileiter $\mathrm{zu}$ arbeiten und gleichzeitig sein juristisches Doktorat vorzubereiten. Im Jahr 1904 promovierte er im Fach Handelsrecht zum Thema des Vergleichs bei Aktiengesellschaften. ${ }^{9}$ Mit Unterstützung seines Vaters entschloß sich Paul Reynaud im Januar 1907 zu einer Weltreise, die ihn in Begleitung eines Freundes durch Mexiko, die USA, Japan, China, Rußland und Deutschland führte. ${ }^{10} \mathrm{Im}$ September des selben Jahres nach Paris zurückgekehrt,

4 "Dossier d'élève. Promotion 1898. Reynaud, Paul“ und „Bulletin de notes de M. Reynaud, Elève de Deuxième Année“ (Ecole des Hautes Etudes Commerciales, Dossier Paul Reynaud).

5 Diese und die folgenden Angaben zur juristischen Laufbahn Paul Reynauds entstammen scinem Personaldossier, das sich im Archiv des Ordre des Avocats à la Cour de Paris (künftig abgekürzt als: Archives de l'Ordre) befindet. Der Archivar der Pariser Anwaltsvereinigung, Herr Yves Ozanam, gewährte mir freundlicherweise in Auszügen Einblick.

- Vgl. hierzu Fernand Payen/Gaston Duveau, Les règles de la profession d'avocat et les usages du barreau de Paris, Paris ${ }^{2} 1926$, S. 153.

7 Vgl. Reynaud, Mémoires I, S. 53.

8 Die „stage“, d. h. der für die Kandidaten des Anwaltsberufes bis heute obligatorische Ausbildungsabschnitt zwischen Universitätsstudium und der Aufnahme in die Anwaltskammer, umfaßte zur Zeit Reynauds einen Zeitraum von drei bis fünf Jahren. Die „avocats stagiaires“ hatten während dieser Phase bereits das Recht, vor Gericht zu plädieren, und waren den „avocats inscrits“ in ihrem Rechtsstatus weitgehend gleichgestellt. Im Gegensatz zu den letztgenannten durften sie ihre anwaltliche Tätigkeit aber vorläufig nur auf Widerruf ausüben. Die „stage“ konnte zur Ableistung des Wehrdienstes oder auch für die praktische Tätigkeit bei einem „avoué “ unterbrochen werden, der angehende Anwalt hatte in diesem Falle aber weiterhin an den berufsspezifischen Ausbildungsveranstaltungen teilzunehmen und durfte darüber hinaus kein Mandat annehmen. Die fakultative Unterbrechung der "stage“ diente für gewöhnlich vor allem dem Sammeln berufspraktischer Erfahrung und dem Anbahnen von Kontakten zu einem künftigen Mandantenkreis (vgl. Payen/ Duveau, Règles, S. 159-187).

9 Paul Reynaud, Des Concordats de Sociétés anonymes après faillite ou liquidation judiciaire, Paris 1904. Die Soutenance der Arbeit erfolgte am 20. Juni 1904.

10 Siehe hierzu den Reisebericht in seinen Memoiren: Reynaud, Mémoires I, S. 62-85. Irrtümlicherweise gibt Reynaud hier 1906 als Reisejahr an. 
betätigte sich Reynaud ein weiteres Jahr als Sekretär unter anderem bei einem am Handelsgericht zugelassenen Anwalt, bevor er mit Wirkung vom 14. August 1908 endgültig in den Anwaltsstand aufgenommen wurde.

Sein bald zutage tretendes rhetorisches Talent trug Reynaud rasch erste Ehren ein und bot ihm Gelegenheit für erste politische Bewährungsproben. Nach einem traditionell seit Beginn des 18. Jahrhunderts einmal jährlich stattfindenden internen Rhetorikwettbewerb wurde er 1910 zum Ersten Sekretär der hochangesehenen „Conférence des Avocats“ gewählt. Die Bedeutung jener auch "Conférence du stage" genannten Generalversammlung der Pariser Anwaltskammer für die Rekrutierung politischer Eliten im Frankreich der Dritten Republik wurde bereits von den Zeitgenossen konstatiert, ebenso wie der Korpsgeist, der die einander ablösenden Generationen von ehemaligen Sekretären - alljährlich wurden 12 neue Würdenträger durch die Versammlung gewählt - verband. ${ }^{11}$ Der Anspruch, eine besondere Berufung zum Eintritt in politische Ämter zu besitzen, war unter den Mitgliedern durchaus nicht unüblich und wurde als Teil einer langen Tradition gesellschaftlich akzeptiert. ${ }^{12}$ Auch Reynaud war die Überzeugung, einer Elite anzugehören, nicht fremd: In einem Artikel zur alljährlichen feierlichen "rentrée“ der Anwaltskonferenz qualifizierte er den Kreis ihrer ehemaligen Sekretäre als „eine Art von Aristokratie" und verwies selbstbewußt auf die Reihe seiner illustren Amtsvorgänger: „Gambetta, Ribot, Poincaré, Millerand, Barthou, Viviani ...“.13 Trotz der unbestreitbaren Verbindungen zur Politik blieben indes öffentliche Auseinandersetzungen mit politischem Hintergrund im "Palais“ eher die Ausnahme. Um so größere Aufmerksamkeit weit über das juristische Milieu hinaus erregte deshalb die öffentliche Diskussion um Reynauds Abschiedsrede als Erster Sekretär, die ihm Gelegenheit bot, sich durch ein weithin konsensfähiges Bekenntnis zum „republikanischen Modell“ gegenüber einer Minderheit konservativer Anwälte zu profilieren.

Neben der turnusmäßigen Vorbereitung von juristischen Diskussionsgegenständen für die wöchentlichen Zusammenkünfte der "Conférence du stage“ gehörte es zu den traditionellen Hauptaufgaben des Ersten Sekretärs, gegen Ende seiner einjährigen Amtszeit eine akademische Lobrede auf ein berühmtes Mitglied der Anwaltskammer oder einen ihrer ehemaligen Präsidenten zu halten. ${ }^{14}$ Paul Reynaud wählte für die feierliche „rentrée“ des Jahres 1911 den republikanischen Politiker René Waldeck-Rousseau als Vortragsthema - eine Wahl, die von einigen der "Action Française“ nahestehenden Anwälten als Provokation verstanden wurde. Man drohte öffentlich, die Laudatio nötigenfalls durch "action directe“ $z u$ verhindern. In Protestbriefen entfaltete man einen Katalog von heftigsten Vor-

11 Vgl. hierzu die Ausführungen, die Fernand Payen und Gaston Duveau in ihrem zeitgenössischen Handbuch zur "Conférence du stage" machen (Payen/Duveau, Règles, S. 184-187, bes. 185).

12 Vgl. hierzu etwa die Äußerung des ehemaligen Ersten Sekretärs des Jahrgangs 1912/13, Marcel Héraud: „Nous avons pour le Parlement une vocation pour ainsi dire congénitale" (Gilles Le Béguec, L'entrée au Palais-Bourbon: Les filières privilégiées d'accès à la fonction parlementaire (1919-1939). Unveröff. Thèse d'Etat, Paris X - Nanterre 1989, S. 707-710, Zitat: S. 708). Le Béguec vergleicht die "Conférence du stage" im Hinblick auf die Rekrutierung des politisch-administrativen Nachwuchses der Dritten Republik mit der „Ecole Normale d'Administration“ und qualifiziert ihre ehemaligen Sekretäre als „élite de la parole publique“ (Ebenda, S. 708).

13 Paul Reynaud, „La rentrée de la Conférence“, in: La Renaissance, 13. 12. 1913.

14 Vgl. Payen/Duveau, Règles, S. 185-187. 
würfen gegen Waldeck-Rousseau, die vor allem auf dessen laizistische Kirchenpolitik und seine Bemühungen zur Bestrafung der Schuldigen an der Dreyfus-Affäre während seiner Zeit als Ministerpräsident zwischen 1899 und 1902 abzielten. Der Sprecher der protestierenden Anwaltsfraktion, Challamel, resümierte: „WaldeckRousseau a déshonoré notre Ordre; son image ne doit pas être placée dans la galerie de nos maîtres". 15

Auf Bitten der Witwe Waldeck-Rousseaus, die sich in einem ebenfalls öffentlich gemachten Schreiben an den Präsidenten der Anwaltsvereinigung gegen die geplante „éloge" aussprach, um zu erwartende Zwischenfälle und die damit verbundene Schädigung des Ansehens ihres verstorbenen Mannes zu verhindern, entzog der "Conseil de l'Ordre“ Reynaud das bereits zugesagte Thema. ${ }^{16}$ Der Beschluß versetzte den Pariser Justizpalast in tagelange helle Aufregung, Solidaritätsbekundungen für Reynaud seitens einer größeren Zahl von Anwälten blieben nicht aus: Ein förmliches Protestschreiben gegen das Redeverbot erhielt innerhalb von wenigen Tagen 224 Unterschriften, darunter diejenigen von René Viviani, Maurice Bokanowski und Léon Bérard. In einem offenen Brief, unterzeichnet unter anderem von Champetier de Ribes, Charles Reibel, Henri Payen, Paul Istel, Alexandre Millerand und Anatole de Monzie, bot die Vereinigung der "Secrétaires et anciens secrétaires de la conférence des avocats" Reynaud an, die Publikation seiner Rede zu finanzieren. ${ }^{17}$ Die gemäßigte Presse sprach von "procédés terroristes" und "tentatives d'intimidation" und publizierte Aufrufe zur Wiederherstellung der "confraternité professionnelle" und der politischen Toleranz innerhalb des Anwaltsstandes. ${ }^{18}$

Nach kurzem Zögern gab Reynaud seinen Verzicht auf die geplante Eloge in der Presse bekannt, nicht ohne jedoch genüßlich die Versuche des "Conseil de l'Ordre" zu schildern, ihn ohne Aufsehen zu einer Themenänderung zu bewegen. ${ }^{19}$ Intern erklärte er sich gegenüber den Vertretern des Conseil bereit, seinen Redeentwurf nicht vor Ablauf von vier Wochen nach der feierlichen "rentrée“

15 „L'éloge de Waldeck-Rousseau“, in: L'Action Française, 12. 7. 1911. Diese Äußerung trug Challamel eine Duellforderung des Schwiegersohns Waldeck-Rousseaus ein (Le Temps, 20. 7. 1911, gibt den Briefwechsel zwischen den Kontrahenten unter der Rubrik „L'éloge de Waldeck-Rousseau et la conférence des avocats" ausführlich wieder). Vgl. auch die ähnlich drastisch formulierten Vorbehalte des Anwalts Olagnier (Brief an Reynaud vom 24. 7. 1911, abgedruckt in: La Patrie, 24. 7. 1911 und L'Eclair, 25. 7. 1911).

16 "L'éloge de Waldeck-Rousseau à la conférence des avocats", in: Le Temps, 21. 7. 1911. Der Brief findet sich abgedruckt in: Le Temps, 20. 7. 1911. In einem Brief an Reynaud vom 19. 7. 1911 bedauerte Madame Waldeck-Rousseau die Ereignisse und bat Reynaud zu einem persönlichen Gespräch (Archives de l'Ordre, Dossier „W.R.“).

17 Zu den beiden Schreiben und den an der Unterschriftenaktion beteiligten Anwälten siehe: „L'éloge de Waldeck-Rousseau. Les couloirs du Palais en ébullition", in: Le Matin, 20.7. 1911 und 21.7. 1911 sowie „A propos de l'éloge de Waldeck-Rousseau“, in: Le Matin, 22. 7.1911.

Raymond Poincaré weigerte sich in einem Brief an Reynaud vom 22.7. 1911, das Schreiben der Sekretärsvereinigung zu unterzeichnen, da damit eine Entscheidung des "Conseil de l'Ordre" in Frage gestellt werde. Er sei indes bereit, sich an der Subskription zugunsten der geplanten Veröffentlichung zu beteiligen (Archives de l'Ordre, Dossier „W.R."). Reynaud kommentierte das Verhalten Poincarés in der Rückschau lakonisch: "Il pensait au bâtonnat.“ (Reynaud, Mémoires I, S. 93).

18 "Au Palais“, in: Le Temps, 21. 7.1911 sowie Le Matin, 22. 7. 1911.

19 Vgl. „L'éloge de Waldeck-Rousseau à la conférence des avocats. Une conversation avec M. Paul Reynaud", in: Le Temps, 22. 7. 1911. Der als Interview inszenierte Artikel basiert auf einem nahezu wörtlich übernommenen handschriftlichen Entwurf Reynauds (AN, 74 AP 6). 
außerhalb des Palais zu veröffentlichen. ${ }^{20}$ Für seine Rede am 2. Dezember 1911 wählte er schließlich das weniger Anstoß erregende Thema „Le procès de Charles Ier d'Angleterre“, nicht ohne jedoch - wiederum als Spitze gegen die Anhänger der Action Française unter seinen Zuhörern gedacht - seine Sympathien für Cromwell und das Prinzip des Königssturzes kundzutun. ${ }^{21}$

Reynauds beabsichtigte Würdigung jenes Politikers der linken Mitte, der für Maurras und seine Anhänger wie kein zweiter die Politik der „défense républicaine" und der anti-kirchlichen Gesetzgebung verkörperte ${ }^{22}$, darf als sein erstes faßbares Bekenntnis zur demokratischen Republik und zur Laizität verstanden werden. Seinem beruflichen und gesellschaftlichen Fortkommen schadete der Vorfall, nach dem er laut eigenem Bekunden in den Kreisen der Pariser Anwaltschaft als „Revolutionär“ gegolten habe ${ }^{23}$, nicht. Nur wenige Monate nach seinem öffentlichkeitswirksamen Eintreten für die Prinzipien der laizistischen Republik und der "défense républicaine" ehelichte er im Februar 1912 die Tochter des späteren Präsidenten der Pariser Anwaltskammer, Henri-Robert. ${ }^{24}$ Reynaud fand damit nicht nur Anschluß an führende Pariser Juristen, sondern trat so auch in enge Beziehung zur Politiker- und Wissenschaftlerfamilie Appell.25

Er galt nun einem weiteren Kreis als vielversprechender Anwalt und Nachwuchspolitiker; die gelegentliche Neigung zur Widerborstigkeit und zum mangelnden Respekt vor Autoritäten sah man ihm nach. Seine Fähigkeit zur prägnanten Formulierung, seine Schlagfertigkeit und der oft beißende Witz, den er zu entfalten verstand, prädestinierten ihn zum rhetorischen „Florettfechter“, als der er ab den zwanziger Jahren parlamentarische Debatten immer wieder zu dominieren verstand. Stets tadellos gekleidet, kleingewachsen und agil, von präziser, aggressiver Intelligenz in der Reaktion auf politische Angriffe - so präsentierte er sich in der Öffentlichkeit. Als Person spaltete er jene, die ihn kannten, in Bewunderer und Skeptiker; gleichgültig ließ er kaum. Die politisch sensiblen Felder, die er betrat, und die oft unpopulären Positionen, die er einnahm, verstärkten diesen Effekt. Man hat Reynaud in der Rückschau mit Edmond Rostands allegorischer Figur des "Chantecler" verglichen. So pathetisch diese Anknüpfung an das Bild des romantischen Helden sein mag, der im Kampf für seine Ideale an der Dummheit und am Egoismus der Umwelt scheitert - ganz von der Hand zu weisen ist sie nicht. Wenig volksnah und vom Wert der eigenen Vorstellungen überzeugt, über-

20 Vgl. die Gesprächsnotiz Reynauds vom 25. 7. 1911 über eine Unterhaltung mit dem scheidenden Präsidenten Busson-Billault und seinem Nachfolger Labori, in dessen Verlauf er von dieser Bedingung des "Conseil de l'Ordre“ unterrichtet wurde (Archives de l'Ordre, Dossier „W.R.“) [Zum Zeitpunkt der Einsichtnahme besaß der Bestand keine eigene Signatur].

21 Reynauds Rede wurde, wie allgemein üblich, auf Kosten der Anwaltsvereinigung gedruckt: Barreau de Paris. Le procès de Charles Ier d'Angleterre. Discours prononcé à l'ouverture de la Conférence des Avocats le 2 Décembre 1911 par M. Paul-Reynaud, Avocat à la Cour d'Appel, Paris 1912 (Archives de l'Ordre, Discours de rentrée des conférences. Avocats stagiaires 1911-1920). Vgl. dazu auch Reynaud, Mémoires I, S. 94.

22 Vgl. Yves-Marie Hilaire, 1900-1945. L'ancrage des idéologies, in: Jean-François Sirinelli (Hg.), Histoire des droites en France. Tome 1. Politique, Paris 1992, S. 519-565, hier: 533.

23 Reynaud, Mémoires I, S. 94.

24 Die imposanten Feierlichkeiten finden sich beschrieben in: Le Cri de Paris, Februar 1912, unter dem süffisanten Titel: „Le Barreau marie sa fille“.

25 Die Ehefrau Henri-Roberts, Jeanne, war die Cousine der Gattin von Paul Appell (1855-1930) (Vgl. Le Béguec, L'entrée, S. 726 f.). 
nahm Reynaud spätestens gegen Mitte der dreißiger Jahre die Dauerrolle des unbequemen Mahners und Krisenanalysten. Sie trug ihn nur, solange er in seiner politischen Position kaum angreifbar war. Als der persönliche Erfolg im Mai/Juni 1940 ausblieb, geriet er ohne größeres Sympathiepolster aus der Bevölkerung zwischen die politischen Fronten, denen er sich in den Jahren zuvor nie eindeutig hatte zurechnen lassen wollen.26

Mit Ausbruch des Ersten Weltkrieges ${ }^{27}$ wurde Reynaud zunächst als Unteroffizier in verschiedenen Unterstützungs- und Transporteinheiten eingesetzt. Nachdem er sich im Verlauf der Schlacht an der Somme 1916 eine Rippenfellentzündung zugezogen hatte, berief man ihn nach kurzem Lazarettaufenthalt in Paris in die "Services de la Présidence du Conseil“" des kurzlebigen Ministeriums Painlevé. Diese Heimatverwendung und der damit verbundene Eintritt in das Milieu der Pariser Ministerialbürokratie waren vermutlich ein Resultat der erworbenen familiären Bande und scheinen auf Vermittlung Emile Borels, des Schwiegersohns von Paul Appell, zustandegekommen zu sein. ${ }^{28}$ Zwischen Juli 1917 und März 1918 beschäftigte sich Reynaud so als Mitarbeiter des späteren Staatspräsidenten Paul Doumer mit der Anwerbung freiwilliger Tschechen, Slovaken und Polen für den militärischen Einsatz gegen die Mittelmächte. Im Rahmen dieser Tätigkeit trat der junge Sous-Lieutenant in engen Kontakt mit der tschechischen Unabhängigkeitsbewegung in Paris und erhielt als Mitglied einer diplomatisch-militärischen Mission bald auch Gelegenheit, an einer Episode in der Geschichte der tschechischen Staatswerdung teilzuhaben, die legendären Ruf erlangte: an der "Anabasis“ der tschechischen Legion in Sibirien. ${ }^{29}$

Mit der Rückkehr nach Frankreich - die für Reynaud ohne erkennbare Wiedereingliederungsprobleme vonstatten ging - machte sich der demobilisierte Leutnant zielstrebig an den Ausbau seiner politischen Karriere, die er bereits vor Beginn seines Kriegseinsatzes vergeblich von der regionalen auf die nationale Ebene zu verlagern versucht hatte. ${ }^{30}$ Nach einem Wahlkampf in seinem Heimatdepartement, den er unter dem Vorzeichen des antibolschewistischen Engagements und der Union aller reformbereiten Kräfte geführt hatte ${ }^{31}$, gelang ihm über eine dem Bloc National nahestehende "Liste d'Union et de Concentration Républicaine“

26 Der "Chantecler“-Vergleich findet sich bei: Violet Trefusis, Prélude au désastre, Paris 1990, S. 1527; stellvertretend für Charakterisierungen aus dem Mitarbeiterkreis: Alfred Sauvy, De Paul Reynaud à Charles de Gaulle. Un économiste face aux hommes politiques 1934-1967. Scènes, tableaux et souvenirs, Paris 1972, S. 13 ff.; Claude Popelin, Arènes politiques, Paris 1974, S. 45-55.

27 Ausführliche Quellen- und Literaturangaben zu diesem wie zu ausgewähiten weiteren Abschnitten seiner politischen Biographie finden sich jeweils in den einschlägigen Kapiteln dieser Arbeit.

28 Borel hatte das Amt des „Secrétaire général de la Présidence“ unter Painlevé von September 1917 bis zu dessen Sturz im November 1917 inne. Vgl. hierzu den aufschlußreichen Bericht der Schriftstellerin Berthe Borel, der Ehefrau Emile Borels, die in ihren unter Pseudonym verfaßten Memoiren über die Umstände der Berufung Reynauds Auskunft gibt: Camille Marbo, A travers deux siècles. Souvenirs et rencontres (1883-1967), Paris 1968, S. 166 f. und 240.

29 Vgl. dazu im einzelnen Kapitel IV dieser Arbeit. Die Angaben zu Reynauds militärischem Werdegang sind seinem Personalakt im französischen Heeresarchiv entnommen: „Etat des Services de M. Reynaud, Jean, Paul“ (Service Historique de l'Armée de Terre (SHAT), Dossier No. 42325: Reynaud, Jean, Paul, Capitaine de réserve du train). Vgl. auch Reynaud, Mémoires I, S. 109 f.

$30 \mathrm{Zu}$ seiner gescheiterten Bewerbung um einen Abgeordnetensitz des Departements Hautes-Alpes im Mai 1914 vgl. Kapitel III.3.b) dieser Arbeit.

31 Vgl. dazu etwa: André Honnorat/Paul Reynaud, „A nos amis, les électeurs de Barcelonnette“, in: Journal de Barcelonnette, 29. 11. 1919 sowie ausführlich unter Kapitel V.1. 
im November 1919 erstmals der Sprung in die Kammer der Abgeordneten. Mit 41 Jahren trat Reynaud damit relativ spät in die "große" Politik ein; nichtsdestoweniger hatte er Anteil an einem allgemeinen Generationswechsel in der politischen Klasse Frankreichs nach dem Ersten Weltkrieg, gehörte er doch jener Generation der zwischen 1871 und 1880 Geborenen an, die - nicht zuletzt bedingt durch die Kriegsverluste unter den jüngeren Jahrgängen - mit den Wahlen von 1919 eine außergewöhnlich dominierende Stellung innerhalb der parlamentarischen Rechten einzunehmen begann. ${ }^{32}$ Als Mitglied der neugegründeten Kammerfraktion der "Action républicaine et sociale“, in der sich Sympathisanten des „Mouvement de la Quatrième République“ mit Vertretern der Rechten und der rechten Mitte zusammenfanden, stand er Maurice Bokanowski, Joseph Barthélemy und Pierre Taittinger nahe. Der neugewählte Abgeordnete, der sich ein Vergnügen daraus machte, von den hintersten Bänken der Kammer aus kritische Bemerkungen über die rhetorischen Qualitäten der Redner mit seinem Nachbarn, dem ebenfalls frisch eingetretenen Deputierten Marcel Plaisant, auszutauschen ${ }^{33}$, begnügte sich nicht lange mit der Rolle des bloßen Beobachters. Galt seine parlamentarische Arbeit zunächst den "großen“ Themen des französischen Liberalismus, wie der „décentralisation" der Staatsverwaltung oder der Reform des parlamentarischen Systems, so kristallisierten sich bald auch persönliche Vorlieben heraus, insbesondere die Außen- und Wirtschaftspolitik. Auf diesen Feldern entfaltete Reynaud in den Jahren seiner Tätigkeit als Parlamentarier und dann als Minister zwischen 1919 und 1924 sowie 1928 und 1940 seine wichtigsten Initiativen, die ihn im Laufe der Zeit in Gegensatz zu allen bedeutenden politischen Strömungen bringen sollten.

Seit 1921 verfügte Reynaud über Sitz und Stimme in der prestigeträchtigen außenpolitischen Kommission der Abgeordnetenkammer, für die er sich unter anderem durch eine erfolgreiche Mission zur Verteidigung französischer Finanzinteressen in Mexiko im Herbst 1920 empfohlen hatte. ${ }^{34}$ Hier wie im Plenum und in der Presse profilierte sich der Wirtschaftsexperte Reynaud als kompetenter und innovativer Kritiker der französischen Deutschland- und Reparationspolitik der frühen zwanziger Jahre. In Opposition zur Politik Briands und gegen den Willen eines Teils der französischen Industrie forderte er ab Anfang 1921 die Regelung der deutschen Reparationsverpflichtungen vorwiegend durch Sachleistungen für den Wiederaufbau der kriegszerstörten nordostfranzösischen Gebiete. Enttäuscht

32 Jean-Luc Pinol, 1919-1958. Le temps des droites?, in: Jean-François Sirinelli, Histoire des droites en France, Paris 1992, S. 291-389, hier: 296-299.

33 Marcel Plaisant, Un portrait inédit de Paul Reynaud, in: Revue politique et parlementaire 68 (1966), S. 38-48.

34 Ende September 1920 war Paul Reynaud, der sich zu diesem Zeitpunkt in Südamerika aufhielt, durch den Quai d'Orsay mit einer halboffiziellen Mission in Mexiko beauftragt worden. Diese hatte den Zweck, im Auftrag eines britisch-französischen Gläubigerkonsortiums die im Verlaufe der mexikanischen Revolution in Frage gestellte Anerkennung der Auslandsverbindlichkeiten des Landes zu sichern. Reynaud führte einige Gespräche mit Staatspräsident Obregon und Außenminister Hidalgo, konnte aber keine festen Zusagen erreichen. Eine ausführliche Dokumentation hierzu findet sich in: Archives du Ministère des Affaires Etrangères (künftig: MAE), B-Amérique, Mexique, 55. Vgl. auch den Bericht des französischen Botschafters in Mexiko an den Quai d'Orsay, 4. 10. 1920 (Centre des Archives Diplomatiques de Nantes (CAD/Nantes), Botschaftsakten Mexiko, 12). 
vom faktischen Scheitern des Wiesbadener Abkommens legte er im Frühjahr 1922 ein neues, aufsehenerregendes Konzept vor, das in bewußter Absetzung von den Schwächen der reparationspolitischen Bestimmungen des Versailler Vertrages neben der Festlegung einer exakten Zahlungssumme die Einhaltung eines bestimmten Zahlungsmodus vorsah, der von der Unmöglichkeit höherer deutscher Leistungen ausging. Im einzelnen sollten durch die Erhöhung des Kapitals deutscher Aktiengesellschaften und die Hypothekenbelastung des deutschen Immobilienbesitzes zugunsten der alliierten Gläubiger regelmäßige französische Reparationseinkünfte garantiert werden. Langfristig erhoffte sich Reynaud darüber hinaus eine gemeinsame Nutzung der Ressourcen beider Länder, insbesondere der französischen Eisenerz- und der deutschen Kohlevorkommen, welche den beiderseitigen Interessen gerecht werden und so die künftigen deutsch-französischen Wirtschaftsbeziehungen in den Rahmen des gesamteuropäischen Wiederaufbaus stellen sollte. Das ehrgeizige Projekt, zu dessen Umsetzung Reynaud Verbindungen zur deutschen Industrie geknüpft hatte, scheiterte am Mißtrauen deutscher Regierungsstellen und am Unwillen Poincarés, sich in der Reparationsfrage auf bilaterale Verhandlungen mit Deutschland einzulassen. ${ }^{35}$

Obwohl sich Paul Reynaud mit seinen Vorstößen deutlich außerhalb seiner eigenen Mehrheit gestellt hatte, brachte die Niederlage des Bloc National bei den Parlamentswahlen vom Mai 1924 auch für ihn das vorläufige Ende seines Abgeordnetenmandats. Als Kandidat einer Liste, die unter der Bezeichnung „Républicains de gauche" antrat, konnte er zwar deutlich mehr Stimmen auf seinen Namen vereinen als noch im Jahre 1919, doch favorisierte die Wählerschaft des Departements Basses-Alpes - entsprechend dem nationalen Trend - eine Liste aus Sozialisten und Radikalen. ${ }^{36}$ Bei Nachwahlen nur wenig später unterlag er erneut einem sozialistischen Mitbewerber. ${ }^{37}$

In der nun folgenden Zeit eines vierjährigen „Exils“ ${ }^{\text {38 }}$ fern des parlamentarischen Mandats wandte sich Reynaud wieder seinem Anwaltsberuf zu und führte daneben bis 1926 einen verbissenen Kampf gegen die Politik des regierenden Linkskartells. In Presseveröffentlichungen und auf Reisekampagnen, die ihn durch ganz Frankreich führten, kritisierte er insbesondere die Innen- und Finanzpolitik des Kartells, dem er falsche Versprechungen und Unfähigkeit vorwarf. ${ }^{39}$ Sein Einsatz, seine beredte Intelligenz und nicht zuletzt sein gutes Verhältnis zu Alexandre Millerand, dessen "Ligue Républicaine Nationale“ den organisatorischen Rahmen für Reynauds Redekampagnen bildete, machten ihn zum geeigneten Kandidaten der „modérés“ für die Nachwahlen, die im März 1926 im zweiten Sektor von Paris stattfanden. Zusammen mit einem Kandidaten der nationalen Rechten, dem Publizisten Henri de Kerillis, trat Reynaud unter einem besonders auf die mittelständische Bevölkerung des Wahlkreises zugeschnittenen Programm

35 Vgl. dazu im folgenden ausführlich Kapitel V.

$36 \mathrm{Zu}$ den aufgeschlüsselten Wahlergebnissen im Departement siehe: „Résultat des Elections du 11 Mai 1924“, in: Journal de Barcelonnette, 29. Juni 1924.

37 Vgl. „Résultats des Elections du 17 Aout 1924“, in: Journal de Barcelonnette, 31. August 1924.

38 Reynaud, Mémoires I, S. 202.

39 Ebenda. Vgl. hierzu seine Stellungnahme im Rahmen einer Umfrage über die Rolle der Opposition nach der Wahlniederlage des "Bloc National": „Après le combat. La Chambre du 11 mai et les devoirs de la minorité", in: Revue Hebdomadaire, 19. 7. 1924, S. 336-344. 
an, mußte sich indes nach dem zweiten Wahlgang knapp einer durch Jacques Duclos geführten kommunistischen Liste geschlagen geben. Der Festigung seiner Position innerhalb der gemäßigten Rechten auch in der Hauptstadt tat die unerwartete Niederlage jedoch keinen Abbruch: Als Kandidat der „Républicains Nationaux" im bürgerlichen 2. Pariser Arrondissement erreichte er bei den Parlamentswahlen vom April 1928 einen überwältigenden Sieg gegen seinen radikalsozialistischen Hauptkonkurrenten und zog erneut in die Deputiertenkammer ein. 40

Mit der 14. Legislaturperiode vollzog sich ein Wechsel im politischen Führungspersonal Frankreichs, in dessen Verlauf altbewährte Politiker wie Briand, Poincaré und Caillaux aus dem öffentlichen Leben zurücktraten und einer neuen Generation Platz machten, zu der neben Pierre Laval, Edouard Daladier, PierreEtienne Flandin und André Tardieu bald auch Paul Reynaud gezählt wurde.41 $\mathrm{Daß}$ dieser sich bis Mitte 1929 als einer der Führer der neuen Mitte-Rechts-Mehrheit in der Kammer profilieren konnte, lag nicht zuletzt an seinem erfolgreichen Engagement für die Ratifizierung des Mellon-Bérenger-Abkommens vom April 1926, das die Rückzahlung der französischen Kriegs- und Nachkriegsschulden gegenüber den USA regeln sollte. Sein Eintreten für eine unzweideutige Erfüllung der französischen Verpflichtungen traf gleichermaßen auf den Widerstand der zögernden Linken um Herriot und Blum wie denjenigen der extremen Rechten. Seine Intervention war mitentscheidend für die Überwindung von Widerständen innerhalb der Regierungsmehrheit und die denkbar knappe Ratifizierung durch die Kammer im Juli 1929.42

Hatte Reynauds deutschlandpolitisches Engagement seit 1923 kaum mehr Aufsehen erregt, so änderte sich das, als im September 1929 bekannt wurde, daß er im April des gleichen Jahres während einer Informationsreise in Deutschland Gespräche nicht nur mit Reichstagsabgeordneten aller Schattierungen, sondern auf Vermittlung des deutschen Industriellen Arnold Rechberg auch mit Vertretern der außerparlamentarischen nationalistischen Rechten und des Stahlhelm geführt hatte. Angesichts einer heftigen Pressekampagne gegen ihn ging er um die Jahreswende 1929/30 selbst zur Offensive über und präsentierte der Öffentlichkeit sein umfassendes Konzept eines "néo-nationalisme“. Er forderte darin die Ergänzung der traditionellen, auf Ausbau der französischen Militärmacht und die Bildung von Allianzen mit osteuropäischen Partnern gerichteten französischen Sicherheitspolitik durch den gezielten Interessenausgleich mit der deutschen Rechten, insbesondere auch mit großindustriellen Kreisen, und plädierte unter anderem für den Verzicht auf eine fortdauernde Besetzung des Rheinlandes. ${ }^{43}$

Andere, technischere Fragestellungen traten mit Beginn seines ersten Ministeramtes im zweiten Kabinett Tardieu, in dem ihm das Finanzressort zufiel, in den Mittelpunkt seiner Tätigkeit. Zwischen März und Dezember 1930 hatte er unter

40 Siehe hierzu im einzelnen Kapitel VI dieser Arbeit.

41 Zum Generationswechsel vgl. Bonnefous, Histoire politique V, S.V.

42 Vgl. zur Debatte und zur Wirkung der Intervention Reynauds: Bonnefous, Histoire politique IV, S. 348-359; eingehender dazu im folgenden Kapitel VII.1.

43 Vgl. dazu die Artikelreihe in der Revue Hebdomadaire vom Januar/Februar 1930, in der er in Auseinandersetzung mit dem Herausgeber der Zeitschrift, François Le Grix, seine Thesen entwickelte (Kapitel VII.2). 
anderem die finanzpolitische Seite eines umfassenden Modernisierungsprogrammes zu vertreten und Strategien gegen die mittlerweile weltweit grassierende Wirtschaftskrise zu entwickeln. Nichts deutete zu diesem Zeitpunkt indessen bereits darauf hin, daß der Politiker sich bis Mitte der dreißiger Jahre zu einem Vertreter ausgesprochen unorthodoxer währungspolitischer Ansichten wandeln würde. Im Januar 1931 übernahm Reynaud das Ressort des Kolonialministers in der ersten Regierung Pierre Lavals und erhielt nach dessen Sturz in der kurzlebigen dritten Regierung Tardieu (Februar - Juni 1932) die Funktion des Justizministers übertragen. ${ }^{4}$

Mit der erneuten Linksverschiebung der seit 1928 bestehenden parlamentarischen Mehrheit als Folge der Wahlen des Jahres 1932 fand sich Reynaud von neuem in der Rolle des Oppositionspolitikers wieder. Mehr als sechs Jahre lang blieb er nun allen Regierungsämtern fern, arbeitete gegen das erneuerte Linkskartell der Jahre 1932 bis 1934 ebenso wie gegen die Volksfront zwischen 1936 und 1938. Fern hielt er sich jedoch auch dem Versuch einer „Union nationale“ unter Gaston Doumergue im Jahre 1934, deren politisches Profil - der Regierung gehörten Vertreter aller gemäßigten Parteien außer den Sozialisten an - eine Teilnahme Reynauds nahegelegt hätte, oder den folgenden rechtsgerichteten Regierungen Flandin und Laval. Stattdessen führte er ab 1934 mehrere verbissene Kampagnen, in denen er teils einschneidende Reformen vorschlug, um der wirtschaftlich prekären Lage, den daraus resultierenden sozialen Unruhen sowie der zunehmenden Kritik am parlamentarischen Regime zu begegnen und nicht zuletzt Frankreichs Außenpolitik auf entschlossenen Widerstand gegen die hegemonialen Ansprüche des deutschen Nachbarn auszurichten. Diese Initiativen sollten ihn in Gegensatz zu nahezu der gesamten politischen Klasse Frankreichs bringen und selbst langjährigen Weggefährten aus dem eigenen Lager entfremden.

So setzte sich Reynaud neben André Tardieu und René Coty in der im März 1934 gegründeten „Commission de la Réforme de l'Etat" für Maßnahmen zur Stärkung der Exekutive und zur effizienteren Parlamentsarbeit ein. Als einer der ersten französischen Politiker verfocht er angesichts der Frankreich benachteiligenden weltwirtschaftlichen Entwicklung seit der Abwertung von englischem Pfund und amerikanischem Dollar ab Juni 1934 öffentlich die Abwertung des Franc unter dessen Goldwert von 1926. Seine Forderung berührte ein Tabuthema und stieß auf entschlossenen Widerstand aus allen Parteien des politischen Spektrums, von Handelskammern und Arbeitgebervereinigungen..$^{45}$ Nahezu parallel hierzu griff Reynaud im Rahmen der Diskussion um die Verlängerung des Militärdienstes, die im Frühjahr 1935 in Öffentlichkeit und Parlament in der Frage einer angemessenen Antwort auf die deutsche Hochrüstung entbrannte, ein weiteres kontrovers diskutiertes Thema auf. In Anlehnung an Vorschläge des damaligen Oberstleutnants de Gaulle und gegen die Vorstellungen führender Militärs wie Pétain, Weygand und Gamelin forderte er - vergeblich - die Schaffung eines autonomen Panzerkorps als schneller Eingreifreserve und zur Untermauerung der französischen Bündnisverpflichtungen gegenüber den östlichen Verbündeten. In

44 Reynaud, Mémoires I, S. 299-337.

45 Julian Jackson, The politics of depression in France 1932-1936, Cambridge 1985, S. 187. 
Opposition zur kompromißbereiten, italienfreundlichen Außenpolitik Lavals während der Äthiopienkrise verweigerte sich Reynaud - wiederum mit Blick auf die von Deutschland ausgehende Kriegsgefahr - Ende 1935 jedem Entgegenkommen an Mussolinis Gebietsansprüche, das Frankreich letztlich von der sanktionswilligen öffentlichen Meinung Großbritanniens trennen und so Hitler in die Hände arbeiten werde. ${ }^{46}$ Sein Engagement, das ihn an die Seite Léon Blums und der Mehrheit der Radicaux gegen nahezu die gesamte Rechte stellte, führte zum Bruch mit André Tardieu. ${ }^{47}$ Ein Jahr später entfremdete sich Reynaud durch sein Eintreten für eine französisch-russische Allianz als Gegengewicht gegen das deutsche Ausgreifen nunmehr dauerhaft weite Teile des eigenen rechten Lagers. 48

Mit dem definitiven Ende der Volksfront und der Rückkehr eines ins bürgerliche Lager übergewechselten Daladier an die Macht erhielt Reynaud im April 1938 die Chance zum Wiedereintritt in die Regierungsverantwortung. Obwohl er bekanntermaßen eine andere Konzeption in Verteidigungsfragen vertrat als Daladier und - wie sich bald zeigen sollte - auch dessen Deutschlandpolitik kritisierte, führte an dem Finanzexperten und mittlerweile führenden Kopf der gemäßigten Rechten kein Weg mehr vorbei. Zunächst auf das zweitrangige Amt des Justizministers beschränkt, übernahm er nach einer Kabinettsumbildung im November 1938 das Finanzressort und zeichnete bald verantwortlich für eine Neuorientierung der französischen Wirtschaft im liberalen Sinne, die von außerordentlicher Tragweite sein sollte. Unterstützt durch einen hochkarätigen Stab junger Mitarbeiter, darunter Alfred Sauvy und Michel Debré, machte Reynaud mittels einer Serie von Dekreten wichtige Errungenschaften der Volksfront, u. a. die VierzigStunden-Woche, weitgehend rückgängig, setzte staatliche Sparmaßnahmen durch und erweiterte Arbeitgeberrechte. Über eine prononcierte Austeritätspolitik erreichte er so bis Sommer 1939 einen bemerkenswerten Wiederaufschwung der französischen Wirtschaft; ein kommunistisch inspirierter Generalstreik hatte Ende November 1938 aufgrund der verbreiteten Sympathie für Daladiers Wiederaufrüstungspolitik in der Bevölkerung und selbst in den Gewerkschaften nur geringe Resonanz gefunden. Mit Ausbruch des Krieges leitete Reynaud die Umstellung der französischen Wirtschaft auf die Kriegsproduktion in die Wege, wobei er auf insgeheim bereits vorbereitete Maßnahmen unter anderem zur Devisenbewirtschaftung zurückgreifen konnte. ${ }^{49}$

Am 20. März 1940 stürzte Daladier, da er es in den Augen der konservativen Abgeordneten und der Öffentlichkeit nicht vermocht hatte, die lastende Atmosphäre der "drôle de guerre" durch einen befreienden Schlag an der Peripherie des europäischen Kriegsgeschehens zu entspannen.50 Am nächsten Tag ernannte

46 Siehe hierzu insbesondere Reynauds Parlamentsrede vom 27. Dezember 1935 (Journal Officiel (künftig: JO), Chambre des Députés, Débats parlementaires, Séance du 27 décembre 1935, S. $2815 \mathrm{f}$.).

$47 \mathrm{Zu}$ den Motiven Tardieus vgl. dessen offenen Brief an Le Temps vom 31. Dezember 1935, in dem er insbesondere seine Freundschaft zu Laval und seine Bewunderung für Mussolini zum Ausdruck brachte.

48 Vgl. dazu unten Kapitel X.1.

49 Ausführlich hierzu Kapitel X.1 dieser Arbeit.

50 Über die entscheidende Debatte berichtet ausführlich: Hans-Jürgen Heimsoeth, Der Zusammenbruch der Dritten Französischen Republik. Frankreich während der „Drôle de Guerre" (1939/ 1940), Bonn 1990, S. 277-279. 
Staatspräsident Lebrun Paul Reynaud zum Nachfolger, der sein Amt unter ungünstigsten Bedingungen antrat. Unbeliebt in der Bevölkerung und ohne großen parlamentarischen Rückhalt, erreichte er bei der Präsentation seiner Regierung aus Radikalsozialisten, Sozialisten und einigen Vertretern der Rechten - die Fédération Républicaine um Louis Marin war aus Protest gegen den Eintritt der Sozialisten ferngeblieben - nur die denkbar knappe Kammermehrheit von einer Stimme. Nicht ohne Bedenken nahm Reynaud sein Amt und die damit verbundene Herausforderung auf entschlossenere Kriegführung an..$^{51}$ Sein Drang zur Aktion erlitt indes rasch gravierende Rückschläge und brachte ihn zunehmend in Gegensatz zu jenen, die im Grunde seine engsten Verbündeten sein sollten - zur eigenen militärischen Führung und zu den britischen Alliierten. ${ }^{52}$ Am 9. Mai kündigte Reynaud seinen Rücktritt an, widerrief ihn aber bereits am folgenden Tag unter dem Eindruck des deutschen Angriffs auf Frankreich. Angesichts des bereits eine Woche später sich abzeichnenden Desasters versuchte er, durch Regierungsumbildungen am 18. Mai und am 5. Juni, in deren Verlauf Weygand und Pétain, schließlich auch de Gaulle in das Kabinett aufgenommen wurden, die Voraussetzungen für eine Konzentration aller Kräfte zu schaffen. Nachdem jedoch im Kabinett eine immer stärker werdende Strömung für einen Waffenstillstand eintrat und ein letzter verzweifelter Plan zur Gründung einer englisch-französischen Staatengemeinschaft keine Mehrheit gefunden hatte, trat Reynaud, der sich nach wie vor für eine Fortsetzung des Kampfes von Afrika aus aussprach, am 16. Juni 1940 von seinem Amt zurück. Zu seinem Nachfolger ernannte Präsident Lebrun noch am selben Tag Pétain, der am 17. Juni ein Waffenstillstandsersuchen an die deutsche Regierung richtete. ${ }^{53}$ Reynaud, der nach einigem Zögern das Amt des Botschafters in den Vereinigten Staaten ablehnte, das ihm Pétain offerierte, wurde im September 1940 verhaftet und zunächst im Pyrenäenfort Portalet interniert. Auf deutsches Verlangen hin im November 1942 ausgeliefert und in den Konzentrationslagern Oranienburg und Sachsenhausen, schließlich zeitweise zusammen mit Gamelin und Weygand auf Schloß Itter in Tirol gefangengehalten, wurde er dort im Mai 1945 von amerikanischen Truppen befreit.

Nach 1945 hatte es Reynaud schwer, in die gewohnt einflußreichen Positionen zurückzukehren, die Wiederherstellung seines beschädigten politischen Rufes gelang ihm ohnehin nie mehr völlig. Dies schloß die Übernahme von Ministerämtern nicht aus: Mit Ausnahme einer einjährigen Tätigkeit als stellvertretender Ministerpräsident in der Regierung seines politischen Freundes aus Vorkriegszeiten, Joseph Laniel (28.6. 1953-13.6. 1954), beschränkten sich seine erneuten Erfahrungen jedoch auf Intermezzi von wenigen Tagen bzw. Wochen in den ephemeren Kabinetten Marie (26. 7.-28. 8. 1948) und Queuille (2. 7.-4. 7. 1950). Das Kriegsende hatte mit der Niederlage des Vichy-Regimes die klassische und die gemäßigte Rechte Frankreichs in ihrer Gesamtheit zumindest vorläufig diskreditiert, und andere Kräfte drängten in den Vordergrund: Der neugegründete christdemokratische MRP und der PCF gingen aus den Wahlen zu den ersten verfassunggebenden

51 Vgl. zu den Umständen der Berufung Reynauds: Ebenda, S. 279 f.

52 Im einzelnen dazu ausführlich und präzise: Ebenda, S. 280-297.

53 Siehe unten Kapitel X.2. 
Versammlungen vom Oktober 1945 und Juni 1946 jeweils als Sieger hervor und etablierten sich zusammen mit der SFIO zunächst als die drei führenden politischen Strömungen. ${ }^{54}$ Aus seinem angestammten Wahlkreis im Herzen von Paris verdrängt, hatte sich Reynaud zum zweitenmal in seinem Leben als "parachuté“ in einem ihm völlig fremden Bezirk zu bewerben und erreichte im Juni bzw. November 1946 als Kandidat von Dünkirchen den Wiedereinzug ins Parlament. 55 Die Kammer- und Gremientätigkeit auf nationaler und internationaler Ebene machte ab nun den Hauptteil seines politischen Lebens aus. Als einer der ersten Vertreter des rechten politischen Spektrums in Frankreich setzte er sich engagiert für die europäische Einheit und ein direkt gewähltes europäisches Parlament ein; im August 1954 gehörte er in der Assemblée Nationale zu den Befürwortern der Europäischen Verteidigungsgemeinschaft. ${ }^{56}$ Auch alte thematische Schwerpunkte hatte er rasch wieder aufgegriffen: So war er in der entscheidenden Parlamentsdebatte vom Juni 1948 wiederum als Exponent einer verschwindenden Minderheit im rechten Lager für die Ratifizierung der „Londoner Empfehlungen“ eingetreten, da er die Eingliederung des neuzugründenden deutschen Staates in ein föderalistisch organisiertes Europa als Lösung des jahrhundertealten deutsch-französischen Antagonismus begriff. 57

Nach 1946, nunmehr als bereits knapp 70jähriger, machte er sich für die Reform der Verfassung der Vierten Republik im Sinne einer Stärkung der Exekutive stark. Das jahrzehntealte persönliche Anliegen, das durch die fehlende Stabilität auch der neuen republikanischen Staatsschöpfung reaktiviert worden war, ließ ihn erneut zum Förderer de Gaulles werden. Enttäuscht wandte Reynaud sich noch in seinen letzten Lebensjahren ab, als er erkennen mußte, daß weder dessen Europapolitik noch die „bonapartistische“ Ausübung der Präsidentschaft seinen politischen Grundsätzen entsprachen. ${ }^{58}$

Am 21. September 1966 starb Paul Reynaud im Pariser Vorort Neuilly.

54 Rémond, Droites, S. 238f.; Jacques Chapsal, La vie politique en France de 1940 à 1958, Paris 1984, S. 159.

55 Tellier, Paul Reynaud, S. 154-158.

56 Alfred Grosser, Frankreich und seine Außenpolitik 1944 bis heute, München/Wien 1986, S. 136 f.; Tellier, Paul Reynaud, S. 190-194 und 227-231. Reynaud veröffentlichte seine Europagedanken in einer programmatischen Schrift: Paul Reynaud, S'unir ou périr, Paris 1951. Vgl. Kapitel XI.3.

57 Vgl. JO, Assemblée Nationale, Débats parlementaires, Séance du 16 juin 1948, S. 3579-3581; zu den Debatten vom 11. bis 16. Juni 1948 vgl. Gerhard Kiersch, Parlament und Parlamentarier in der Außenpolitik der IV. Republik, Diss. Berlin 1971, S. 193-206.

58 Jean Lacouture, De Gaulle. Band 2: Le Politique 1944-1959, Paris 1985, S. 555-557 sowie Ders., De Gaulle. Band 3: Le Souverain 1959-1970, Paris 1986, S. 338 und 565 ff., bes. S. 581, 585 f. Seine Kritik an de Gaulles Außenpolitik faßte Reynaud zusammen in: Paul Reynaud, La politique étrangère du Gaullisme, Paris 1964. 